\title{
Feasibility of Machine Learning Algorithms for Predicting the Deformation of Anodic Titanium Films by Modulating Anodization Processes
}

\author{
Sung-Hee Kim ${ }^{1, \dagger}$ and Chanyoung Jeong ${ }^{2, *, \dagger}$ \\ 1 Department of Industrial ICT Engineering, Dong-eui University, 176 Eomgwang-ro, Busanjin-gu, \\ Busan 47340, Korea; sh.kim@deu.ac.kr \\ 2 Department of Advanced Materials Engineering, Dong-eui University, 176 Eomgwang-ro, Busanjin-gu, \\ Busan 47340, Korea \\ * Correspondence: cjeong@deu.ac.kr \\ + These authors contributed equally to this work.
}

check for updates

Citation: Kim, S.-H.; Jeong, C. Feasibility of Machine Learning Algorithms for Predicting the Deformation of Anodic Titanium Films by Modulating Anodization Processes. Materials 2021, 14, 1089. https://doi.org/10.3390/ma14051089

Academic Editors: Stefano Lettieri

Received: 17 January 2021

Accepted: 21 February 2021

Published: 26 February 2021

Publisher's Note: MDPI stays neutral with regard to jurisdictional claims in published maps and institutional affiliations.

Copyright: (c) 2021 by the authors. Licensee MDPI, Basel, Switzerland. This article is an open access article distributed under the terms and conditions of the Creative Commons Attribution (CC BY) license (https:/ / creativecommons.org/licenses/by/ $4.0 /)$.

\begin{abstract}
This study aims to demonstrate the feasibility of applying eight machine learning algorithms to predict the classification of the surface characteristics of titanium oxide $\left(\mathrm{TiO}_{2}\right)$ nanostructures with different anodization processes. We produced a total of 100 samples, and we assessed changes in $\mathrm{TiO}_{2}$ nanostructures' thicknesses by performing anodization. We successfully grew $\mathrm{TiO}_{2}$ films with different thicknesses by one-step anodization in ethylene glycol containing $\mathrm{NH}_{4} \mathrm{~F}$ and $\mathrm{H}_{2} \mathrm{O}$ at applied voltage differences ranging from $10 \mathrm{~V}$ to $100 \mathrm{~V}$ at various anodization durations. We found that the thicknesses of $\mathrm{TiO}_{2}$ nanostructures are dependent on anodization voltages under time differences. Therefore, we tested the feasibility of applying machine learning algorithms to predict the deformation of $\mathrm{TiO}_{2}$. As the characteristics of $\mathrm{TiO}_{2}$ changed based on the different experimental conditions, we classified its surface pore structure into two categories and four groups. For the classification based on granularity, we assessed layer creation, roughness, pore creation, and pore height. We applied eight machine learning techniques to predict classification for binary and multiclass classification. For binary classification, random forest and gradient boosting algorithm had relatively high performance. However, all eight algorithms had scores higher than 0.93, which signifies high prediction on estimating the presence of pore. In contrast, decision tree and three ensemble methods had a relatively higher performance for multiclass classification, with an accuracy rate greater than 0.79 . The weakest algorithm used was k-nearest neighbors for both binary and multiclass classifications. We believe that these results show that we can apply machine learning techniques to predict surface quality improvement, leading to smart manufacturing technology to better control color appearance, super-hydrophobicity, super-hydrophilicity or batter efficiency.
\end{abstract}

Keywords: titanium oxide; systematic surface control; machine learning; nanostructure prediction; anodization

\section{Introduction}

Nowadays, ordered titanium dioxide $\left(\mathrm{TiO}_{2}\right)$ nanotube arrays obtained by $\mathrm{Ti}$ anodization have overwhelmingly attracted scientific and technological interests because of their functional properties. Among its various applications, $\mathrm{TiO}_{2}$ plays a pivotal role because of its chemical stability, nontoxicity, and biocompatibility [1-3]. For example, $\mathrm{TiO}_{2}$ is used in solar cells production [3], photocatalytic processes [4], and self-cleaning coatings [2,5]. It also has antibacterials properties [6] and can be used in semiconductors [2]. $\mathrm{TiO}_{2}$ is also considered a synthetic bone graft substitute $[7,8]$. Considering their superior features including unique structure, high specific surface area, and quantum confinement effect, $\mathrm{TiO}_{2}$ nanotubes/nanoporous arrays are the most frequently fabricated nanostructures. Therefore, various methods such as template-assisted sol-gel synthesis, play a crucial role in the fabrication of $\mathrm{Ti}$ nanostructures $[9,10]$. However, highly aligned nanotubes/nonporous 
structures have increasingly focused on the anodization of Ti. Anodization is a simple electrochemical process performed to produce thick nanoporous/tubular metal oxide and subsequently precious such as $A l, T i$, and $M g$ [11,12]. In general, the majority of principles that apply to the electrochemical anodization of $T i$ in electrolytes containing fluoride ions is the most common method used to create self-organized nanotube arrays [13-15]. The anodic formation of $\mathrm{TiO}_{2}$ is associated with different parameters, which affect the surface morphology and quality of the structure [16-18]. For example, by controlling anodization parameters, such as electrolyte type, electrolyte composition, $\mathrm{pH}$, applied voltage and potential difference, temperature and anodization duration, nanostructures with different morphologies and characteristics can be obtained $[14,15,19,20]$. However, combining all these parameters during anodization to observe all types of surface characteristics is costly. It would be helpful if we can narrow the experimental procedures to a certain range by predicting the core surface factors, which we aim to determine in our present study. Herein, we focused to fabricate nanoporous/nanotubes anodic titanium oxide layers through a one-step anodization process performed in fluoride-containing ethylene glycol at various anodization and voltages. We mainly investigate the influence of applied voltage and duration difference on the growth of $\mathrm{TiO}_{2}$ nanostructures. Based on the systematic control over the two parameters, we obtained 100 samples and categorized surface characteristics from the surface image. We investigated whether we could apply machine learning algorithms to predict an anodic formation of $\mathrm{TiO}_{2}$ structure. We clearly aimed to predict surface characteristics that were classified into two or four groups based on pore structures. Besides the common parameters assessed in $\mathrm{TiO}_{2}$ structures such as surface thickness, these oxide structures are considered significant because they are superhydrophilic, superhydrophbic, and have excellent color appearance on a Ti surface. We believe that if we are able to determine certain parameters of the pore structures without conducting labor-intensive experiments, the time and cost of conducting several experiments can be sufficiently utilized. In this study, a total of 100 samples were produced, and this sample size is relatively small when assessing the feasibility of machine learning algorithms compared to other domains. However, if we can determine the feasibility of predicting surface characteristics, we will be able to harness the appropriate predictive model that should be used to estimate generated surface characteristics, allowing us to focus more on higher granularity of experimental conditions to generate the desired surface characteristics.

Machine learning is a type of artificial intelligence algorithm that uncovers patterns in large datasets using computer-based statistical models. Recently, machine learning has developed a wide range of algorithms, which can be roughly described in three ways: (1) supervised learning, where input and output variables are given and the model is determined from the labeled input dataset; (2) unsupervised learning where a selforganized learning method determines unknown patterns in a dataset without pre-existing labels; and (3) reinforcement learning, where an intelligent agent is defined and interacts with its environment by performing actions and learning from errors or rewards, termed as a trial-and-error approach for learning [21].

Classification is a type of supervised learning technique that categorizes data from prior information. The algorithm is used to predict a discrete value that is assigned to a particular class or group. Imagine a set of photographs of animals where each photo is labeled as a cat, rabbit or other animals. When a new image is assigned, the algorithm has to classify it into one of these labeled categories. Each testing instance is matched with a category, which we call labeled data and is used for training. Classification is performed in two phases, and the labeled dataset is divided into training and test dataset. First, a classification algorithm updates its model with the training dataset, and the analytical model extracted is validated against a labeled test dataset to calculate the model performance and accuracy. Compared to different applications such medical imaging detection or quality control in manufacturing, materials science has recently applied these machine learning techniques considering the difficulties they faced in collecting a large sample of data. However, the prerequisite for machine learning is the existence of prior data. To 
obtain thousands of input data with traditional experimentation methods, a significant amount of cost, time, and effort is required in materials science. Therefore, current research in materials science primarily aims to discover and design new materials by using public datasets on material properties [22]. In our current study, we aim to evaluate and compare eight machine-learning techniques in predicting the experimental result on $\mathrm{TiO}_{2}$ structures, such as pore structure and thickness, by performing different anodization processes.

\section{Experimental Dataset Development}

We degreased the Ti sheets by sonicating in a solution of acetone, ethanol, and deionized (DI) water for $30 \mathrm{~min}$. Subsequently, we applied two different polishing methods. Electrochemical polishing was performed in a mixture containing acetic acid, sulfuric acid and hydrofluoric acid (60:15:25 in volume) at a constant current density of $140 \mathrm{~mA} / \mathrm{cm}$ and a temperature of $20^{\circ} \mathrm{C}$ for $1 \mathrm{~min}$. Chemical polishing was performed by dipping $\mathrm{Ti}$ samples into a stirred mixture of hydrofluoric acid and nitric acid (1:3 in volume) for $10 \mathrm{~s}$. Subsequently, the samples were rinsed with water and ethanol and dried in the air. A combined pretreatment method was conducted by electrochemical polishing followed by chemical polishing. The polished Ti samples were prepared via one-step anodization in an electrolyte of ethylene glycol containing $0.25 \mathrm{wt} \% \mathrm{NH}_{4} \mathrm{~F}$ and $2 \mathrm{wt} \% \mathrm{DI}$ water at $0{ }^{\circ} \mathrm{C}$. The process was performed at $10 \mathrm{~V}$ intervals from $10 \mathrm{~V}$ to $100 \mathrm{~V}$ in a two-electrode cell, with polished Ti samples as anodes and platinum as a cathode. The duration of anodization was at $1 \mathrm{~min}$ intervals from $1 \mathrm{~min}$ to $10 \mathrm{~min}$. Structural and morphological characterizations were executed using a field emission scanning electron microscope (FE-SEM). The structural features and thickness of anodized samples were examined directly from FE-SEM images by Image J software.

\section{Machine Learning Algorithm Development}

\subsection{Data Preprocessing for Classification}

The data were categorized based on the characteristics of surface morphology. We introduced the methodology for the multiclass and binary class categorization on the obtained data. Initially, the surface was labeled into four groups based on the images obtained from FE-SEM images. The second author designed the coding scheme based on the formation of an additional layer, roughness with the hexagonal pattern and pore formation. Each aspect is built upon another, for example, a pore would be created after a layer was formed. Both authors used the scheme and independently coded the same 100 images. The inter-rater reliability was found to be good with kappa $=0.94$. The two raters slightly modified the coding scheme after validation. The four classes are described below (see Table 1 for examples):

- Class 0: oxide layer creation

- Class 1: oxide layer creation with roughness

- Class 2: oxide layer creation with pore creation

- Class 3: oxide layer creation with uniform pore generation

After categorizing the four classes, the images were classified into two categories, according to the presence of the pore structure: "with pore" and "without pore." Without pore category includes Classes 0 and 1, and with pore category includes Classes 2 and 3. A categorization into four classes is advantageous because it results in more granularity; however, because of the small dataset size, we also wanted to test binary classification. The presence of the pore is actually the key aspect we aim to determine considering that when the structure becomes uniform with certain height, it contributes to certain characteristics such as color appearance, super-hydrophobicity or super-hydrophilicity. 
Table 1. Definition of four classes of pore structure and sample images.

\begin{tabular}{|c|c|c|c|c|}
\hline \multirow{2}{*}{$\begin{array}{l}\text { Binary Class } \\
\text { Multiclass }\end{array}$} & \multicolumn{2}{|c|}{ Without Pore } & \multicolumn{2}{|c|}{ With Pore } \\
\hline & Class 0 & Class 1 & Class 2 & Class 3 \\
\hline $\begin{array}{l}\text { Definition } \\
\text { Layer Creation } \\
\text { Layer with Roughness } \\
\text { Pore Creation } \\
\text { Pore with Certain Height }\end{array}$ & Only layer & Layer with roughness & Unstable pore & Uniform pore \\
\hline Sample Image & $\overline{2}$ & sim & 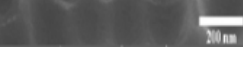 & \\
\hline Thickness(nm) & $76.48 \pm 32.90$ & $147.89 \pm 53.86$ & $267.65 \pm 84.99$ & $819.60 \pm 544.35$ \\
\hline \# of samples & 50 & 14 & 13 & 23 \\
\hline
\end{tabular}

\subsection{Classification Algorithms}

We performed the classification task with eight well-known machine learning algorithms. We aimed to compare the prediction capability of eight algorithms, namely, logistic regression (LogReg) [23], naïve Bayes (NB) [24], k-nearest neighbors (KNN) [25], support vector machines (SVM) [26], random forest (RF) [27], bagging, gradient boosting tree (GBT) [28,29], and decision tree (DecTree) [30]. Here the algorithms and importance of their comparison in this study were briefly explained [31]. LogReg has been widely used for decades even before the advancement of machine learning. It is one type of regression analysis that is conducted when the dependent variable is dichotomous. NB is a simple classification algorithm calculating the conditional probabilities of each input value given in each class value. It is based on strong assumptions about the independence of each input variable. However, it is shown to be effective in many problems. The KNN algorithm is a simple and lightweight supervised machine learning algorithm and it assumes that similar things exist close to one another. It is a nonparametric approach and is stable and robust for small sets and lower dimensional data. The SVM algorithm is used to determine a hyperplane in an $\mathrm{N}$-dimensional space and robustly classifies the data points. It shows great performance when the number of features is less. The DecTree algorithm is used to create a training model that can be used to make decisions on the class by learning simple decision rules inferred from prior data. Decision trees classify the data by starting from the root and compare down to the next leaf/terminal node, with the leaf/terminal node providing the classification of the new data. For multiclass classification problem, the problem can be solved by naturally extending the binary classification techniques when applying the above algorithms [32]. A machine learning paradigm named ensemble learning is widely used where multiple models often called "weak learners", are trained to solve a certain problem and combined to achieve better results. The main hypothesis of ensemble learning is that combining weak learners can obtain more robust and accurate models. RF, bagging and GBT are all ensemble methods. $R F$ is an example of ensemble learning and the logic is simple but powerful if high nonlinearity and complex relationship between dependent and independent variables are observed. Bagging enables the weak learners to learn independently and combines them following deterministic averaging processes. GBT trains many models in an additive manner and is based on the intuition 
that when the next model is combined with the previous models, the overall prediction error is minimized.

\subsection{Performance Measures for Machine Learning Algorithms}

\subsubsection{Binary Classification}

To calculate the performance of the machine learning models, we used K-fold crossvalidation because it is suitable for a dataset with limited sizes [33]. Cross-validation is widely used when we have limited data samples and is a resampling procedure to evaluate machine learning algorithms. To obtain the classification accuracy which is a common practice, 10-fold cross validation was used [34]. It is largely applied because it results in a less biased estimate than simply splitting the dataset into train and test groups. The core idea is to shuffle the dataset randomly; separate a subset of data for validation and use the rest to train a model; and use the left-out data to predict. This process is repeated several times and leaves out a different subset of the data for validation until all the given data is used for learning.

For binary classification, we used area under the receiver operating characteristic (ROC) curve (AUC) [35]. We will first introduce other common measures. For a binary classification problem, accuracy is the proportion of correctly classified instances; however, it is a poor measure for an imbalanced dataset (where $T P=$ true positive, $T N=$ true negatives, $F P=$ false positives, and $F N=$ false negatives.)

$$
\text { Accuracy }=\frac{T P+T N}{T P+T N+F P+F N}
$$

Precision and recall are additional ways to assess the results by breaking down the accuracy formula. Precision quantifies the number of classes predicted positive that actually belong to the positive class. Precision is a good performance measure to apply when the costs of false positive are high.

$$
\text { Precision }=\frac{T P}{T P+F P}
$$

Recall quantifies how many of the actual positives our model captures through classifying it as positive (true positive). It is a good performance measure when there is a high cost associated with false negative.

$$
\text { Recall }=\frac{T P}{T P+F N}
$$

$F 1$ score balances both precision and recall in one number. It is the weighted average of precision and recall. Therefore, this score concerns both false positive and false negative into account. F1 score is usually more useful than accuracy, especially when you have an uneven class distribution.

$$
F 1=2 \times \frac{\text { Precision } \times \text { Recall }}{\text { Precision }+ \text { Recall }}
$$

ROC curves shows the trade-off between the true positive rate (synonym for recall) and false positive rate for the extracted model using different probability thresholds. If we lower the classification threshold, the model classifies more items as positive, thus increasing both false positives and true positives. AUC stands for "area under the ROC curve." The AUC measures the entire two-dimensional area below the ROC curve from $(0,0)$ to $(1,1)$, which means that AUC provides an indication of how good or bad our classifier is performing across all possible classification thresholds. Therefore, AUC ranges in value from 0 to 1 . If the classifier is perfect, then the AUC score is 1.0. If the predictions are $100 \%$ wrong, then the AUC is 0 . The advantage of applying the AUC measure is that it is invariant of data imbalance [36]. For these reasons, we used AUC to estimate the performance of our experiments. 
We used the AUC value as the performance measure. A high AUC value greater than 0.8 denotes a reasonably good prediction rate [37], while an AUC value of 0.5 denotes the predictability of a purely random guess such as flipping a coin.

\subsubsection{Multiclass Classification}

When the number of classes is high, it becomes more complicated to measure the performance. The combinations of true and false classifications for each class increases. Therefore, micro- and macro-averages are calculated for precision and recalls [38]. However, their interpretation differs as these micro- and macro-averages compute slightly different things. In a multi-class classification problem, micro-averaged precision and recall equations are as below where $c$ is the class label.

$$
\begin{gathered}
\text { MicroPrecision }=\frac{\sum_{c} T P_{c}}{\sum_{c} T P_{c}+\sum_{c} F P_{c}} \\
\text { MicroRecall }=\frac{\sum_{c} T P_{c}}{\sum_{c} T P_{c}+\sum_{c} F N_{c}}
\end{gathered}
$$

In multi-class classification, the count of all false instances is as below (Equation (7)), therefore micro-precision and micro-recall are the same. In other words, every single false prediction will be a false positive for a certain class, and every single negative prediction will be a false negative for a certain class. As accuracy is a harmony mean of precision and recall, we report the accuracy.

$$
\sum_{c} F P_{c}=\sum_{c} F N_{c}
$$

A macro-average computes the metric independently for each class and then calculates the average by treating all classes equally. On the other hand, a micro-average computes the average aggregating the contributions of all classes. If there is class imbalance, macro average will have lower values. However, it actually indicates the overall accuracy for all classes, that is, predicting each class is equally important; therefore, we also report the macro-average values.

\section{Results}

\subsection{Experiment Results}

Nanoporous anodic Ti oxide layers are fabricated by a one-step anodization process. In the present study, it is evident that the dimension of the nanostructure, such as thickness, strongly depends on the anodization conditions used. The key factors are the anodization time and voltage. After some preliminary experiments, considering duration and voltage, the formation of the nanostructure is possible in a mixture of ethylene golycol/DI water $/ \mathrm{NH}_{4} \mathrm{~F}$ in a duration ranging from $10 \mathrm{~V}$ to $100 \mathrm{~V}$ and a duration ranging from $10 \mathrm{~min}$ to $100 \mathrm{~min}$. The association between the voltage and time on the thickness of the $\mathrm{TiO}_{2}$ films formed on polished Ti samples was observed by FE-SEM. Table 2A-J shows the surface of $\mathrm{TiO}_{2}$ produced by anodization at different voltages and times. Table $2 \mathrm{~A}-\mathrm{J}$ shows three types of structures-a non-nanoporous structure on polished titanium, a nanoporous structure, and a nanotubular structure, where structure shape increases with anodization voltage and time. Initially, the films are significantly thin with the non-porous layer. The films produced with the lowest voltage (up to $50 \mathrm{~V}$ ) are not ordered, comprising small pores or others without pores. The above mentioned case is not observed, however, in the anodization process under a relatively lower duration ( 1 or $2 \mathrm{~min}$ ). The formation of pores is not created and irregular. The formation of pores is not evident at an anodization duration of $1 \mathrm{~min}$, and the nanoporous structure at an anodization duration of $2 \mathrm{~min}$ could be seen above the applied voltage of $90 \mathrm{~V}$ as shown in Table 2A,B. From the results presented in Table 2F-J, when the higher voltage and duration (greater than $50 \mathrm{~V}$ and $5 \mathrm{~min}$ ) of anodization are used, the $\mathrm{TiO}_{2}$ structures have significant $\mathrm{TiO}_{2}$ nanotube and display more uniform films. As can be seen, with increasing anodization time and applied voltage, the rate of oxide 
growth increases and the $\mathrm{TiO}_{2}$ films become thicker. As anodization of $\mathrm{Ti}$ procedure is performed, metal ions $\left(\mathrm{Ti}^{4+}\right)$ migrate from metal to the oxide/metal interface and dissolve into the solution. At the same time, the oxygen-containing ions $\left(\mathrm{O}^{2-}\right)$ are generated at the oxide/electrolyte interface by the field-enhanced dissolution of $\mathrm{H}_{2} \mathrm{O}$ or $\mathrm{OH}^{-}$under the influence of the electric strength (reactions of 8 and 9) [39].

$$
\begin{gathered}
\mathrm{Ti} \rightarrow \mathrm{Ti}^{4+}+4 e^{-} \\
\mathrm{Ti}^{4+}+2 \mathrm{H}_{2} \mathrm{O} \rightarrow \mathrm{TiO}_{2}+4 \mathrm{H}^{+}
\end{gathered}
$$

It is clear that the thickness of the grown oxide is closely related to the operating condition during anodization. The effective current efficiency of the formation of $\mathrm{TiO}_{2}$ in ethylene glycol-based electrolyte is nearly $100 \%$. We can expect that the thickness of the grown oxide is proportional to the electric charge exchanged from electrolyte during anodization process. In other words, the anodizing potential difference by changing current density, anodizing time, and applied voltage determines the thickness of $\mathrm{TiO}_{2}$. Figure 1 shows the results of thickness for Ti anodization in ethylene glycol-based electrolyte. As can be expected, significantly thicker oxide films are created at higher anodizing duration and applied voltage. At relatively higher voltages (greater than $50 \mathrm{~V}$ ), however, it can be observed that the growth of thickness decreases at specific anodization times. It was considered that the decreasing electrolyte viscosity on the rate of oxide growth in the diffusion during anodization performed in ethylene glycol-based electrolyte. The steadystate oxide growth is a dynamic equilibrium between the rate of oxide growth and the rate of oxide etching at the oxide bottoms. During the oxide growth (which formed metal ions), $\mathrm{pH}$ at the oxide bottoms decreases due to reaction 10 [40].

$$
\mathrm{Ti}+2 \mathrm{H}_{2} \mathrm{O} \rightarrow \mathrm{TiO}_{2}+4 \mathrm{H}^{+}+4 e^{-}
$$

In contrast, decreasing $\mathrm{pH}$ at the oxide bottoms encourages the etching of oxide at the oxide film in the ethylene glycol-based electrolyte containing $\mathrm{H}_{2} \mathrm{O}$ and $\mathrm{NH}_{4} \mathrm{~F}$ (reaction 11) [41].

$$
\mathrm{TiO}_{2}+6 \mathrm{HF} \rightarrow\left[\mathrm{TiF}_{6}\right]^{2-}+2 \mathrm{H}_{2} \mathrm{O}+2 \mathrm{H}^{+}
$$

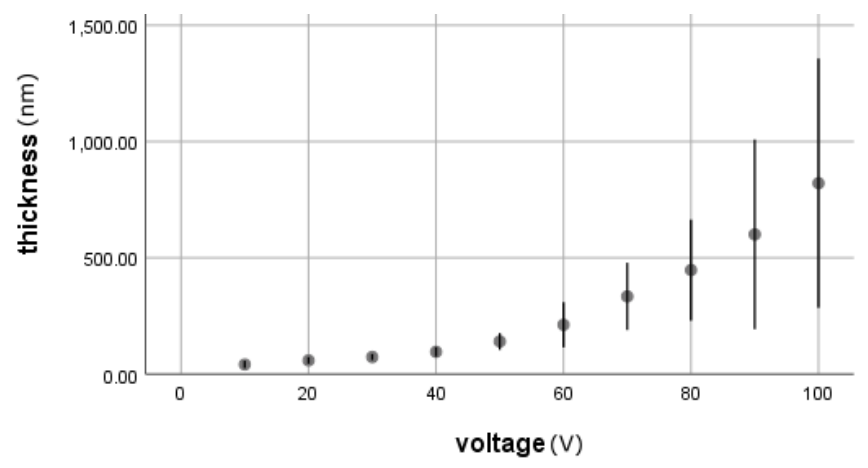

(a)

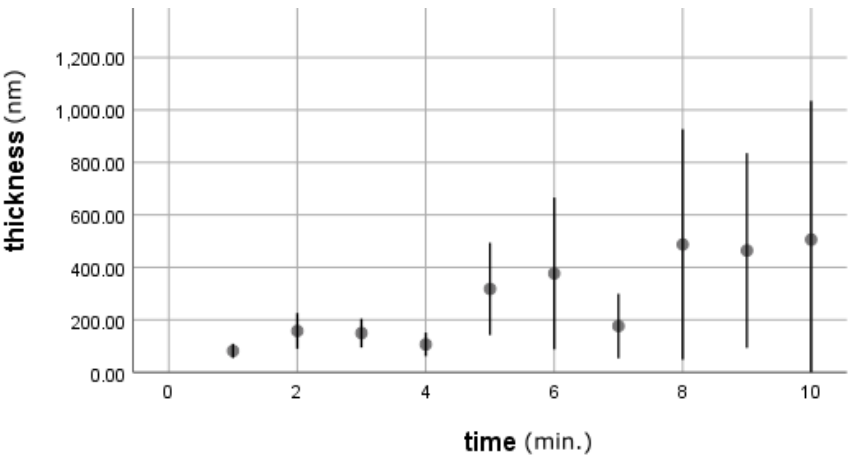

(b)

Figure 1. (a) Mean thickness by voltage from $10 \mathrm{~V}$ to $100 \mathrm{~V}$ (b) Mean thickness by time from $1 \mathrm{~min}$ to 10 min (line indicates $95 \% \mathrm{CI})$. 
Table 2. Cross-sectional view of field emission scanning electron microscope (FE-SEM) images of $\mathrm{TiO}_{2}$ films formed in the anodization performed at $10-100 \mathrm{~V}$ at the anodization time of $1-10 \mathrm{~min}$.

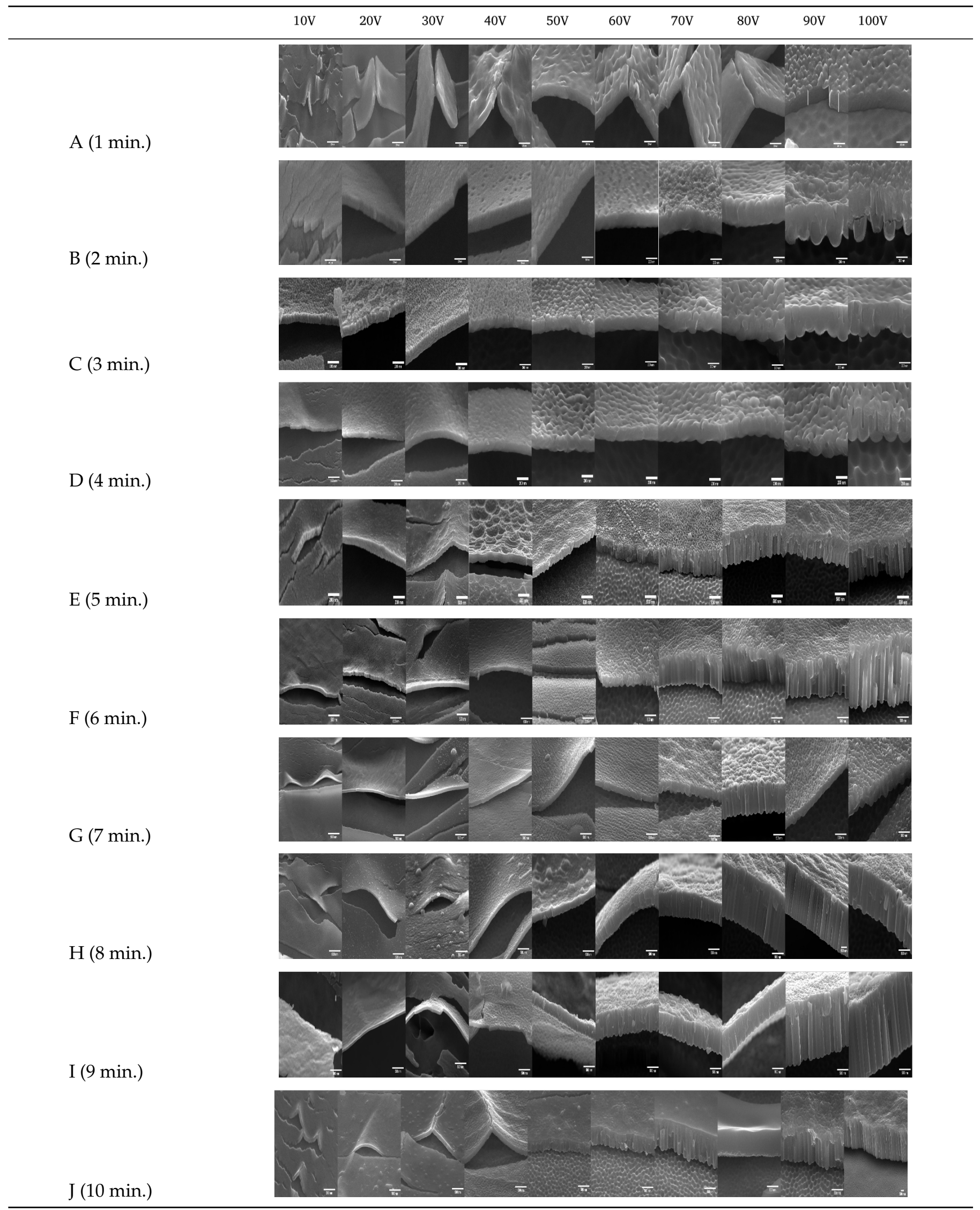


Moreover, it can be attributed to the effect of enhanced hydrolysis of metal ions $\left(\mathrm{Ti}^{4+}\right)$ that appear in the electrolyte as a result of the increasing rate of the oxide growth at higher voltage and time. The hydrolysis consequence in the deposition of the hydrous Ti oxide on the $\mathrm{TiO}_{2}$ surface. Consequently, the thickness is reduced by the deposition. Therefore, the rate of deposition not only the hydrolysis is greater than the rate of chemical etching of the $\mathrm{TiO}_{2}$ layer.

\subsection{Change of Thickness}

Figure 1 shows the mean and variation of thickness by voltage and time separately. Overall, as the voltage and time increase, the mean thickness shows a trend to increase. However, the variation also increases in a nonlinear manner. Compared to voltage change, the fluctuation of the thickness is larger with the time factor. Based on these results, time is a more important factor for oxide thickness change than voltage. Therefore, experiments have shown that time regulation of anode oxidation is a more important factor. Therefore, predicting the anode oxidation time range to obtain the oxide thickness targeted by artificial intelligence can save process time. The following section contains visualizations to help understand the experimental results and to visually inspect how the experimental settings affect the surface and also the relationship by labels that were used for classification. Figure 2 plots the mean thickness by each level of time and voltage that were used in the experiment. The size of the circle indicates the thickness mean.

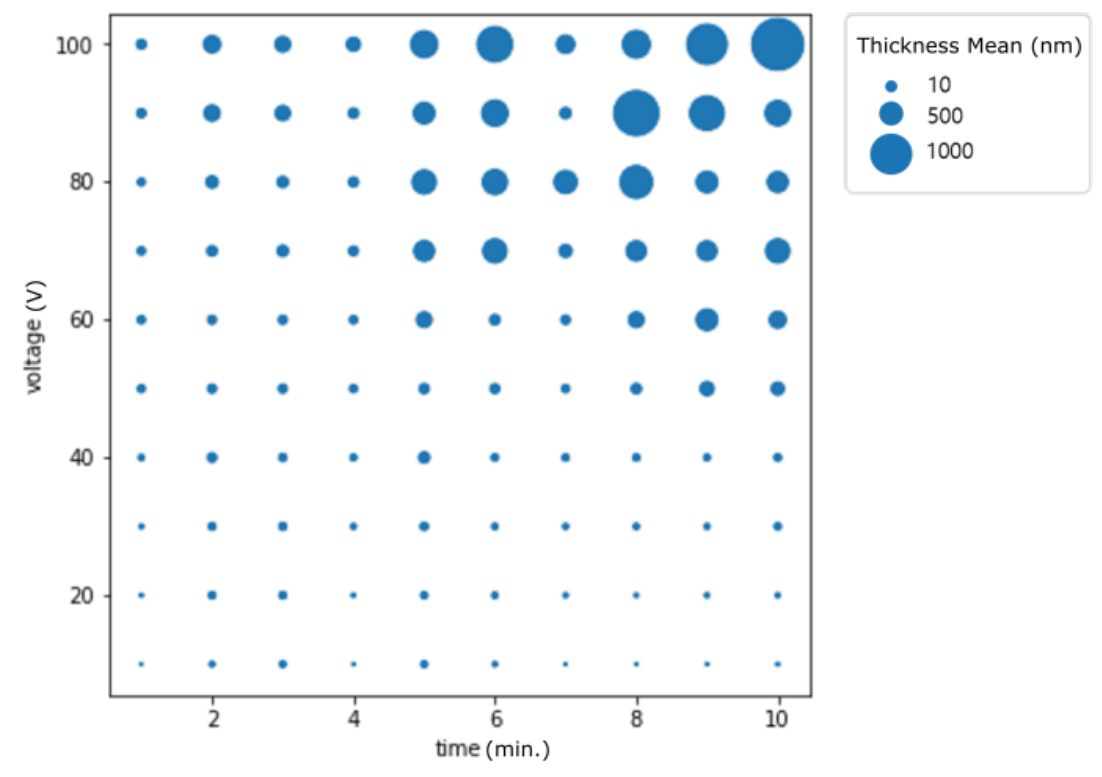

Figure 2. Mean thickness change by voltage and time variation.

Next, we visualized the thickness by experimental settings and separated them by the labels we used for classification to understand the thickness difference among each classification group. Figure 3 presents the binary classification and Figure 4 presents the multi-class classification. For the binary classification, we can observe that surfaces without pore structure are thinner than the surfaces with pore structure. 


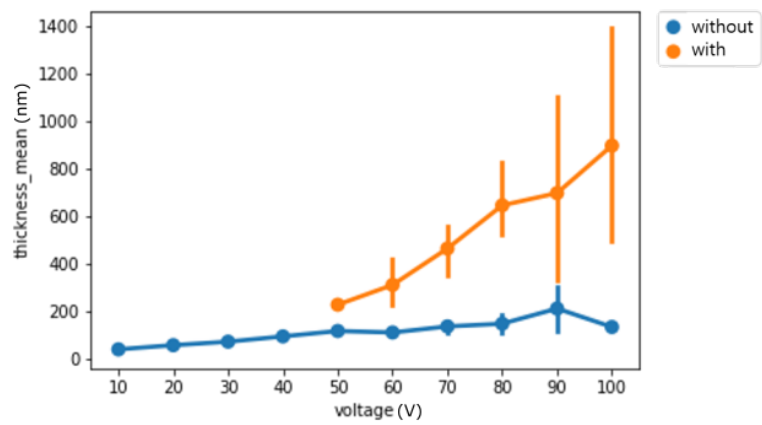

(a)

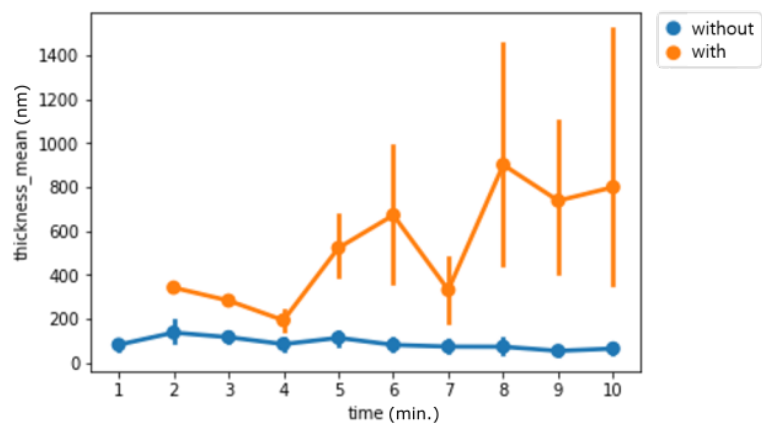

(b)

Figure 3. (a) Binary classification result by voltage from $10 \mathrm{~V}$ to $100 \mathrm{~V}$ (b) Binary classification result by time from $1 \mathrm{~min}$ to $10 \mathrm{~min}$ (line indicates $95 \% \mathrm{CI}$ ).

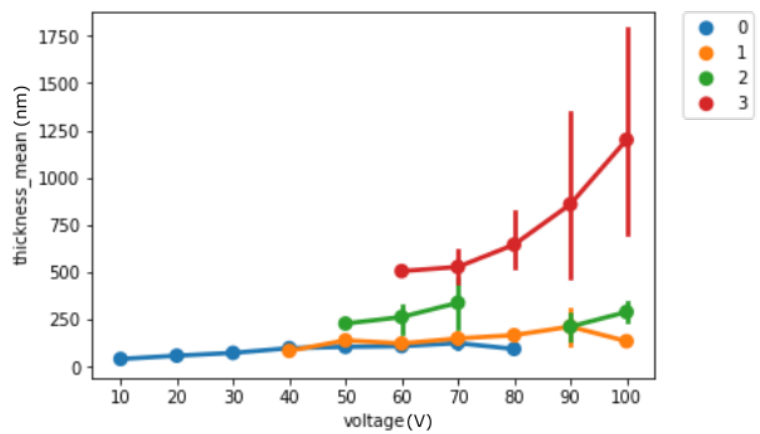

(a)

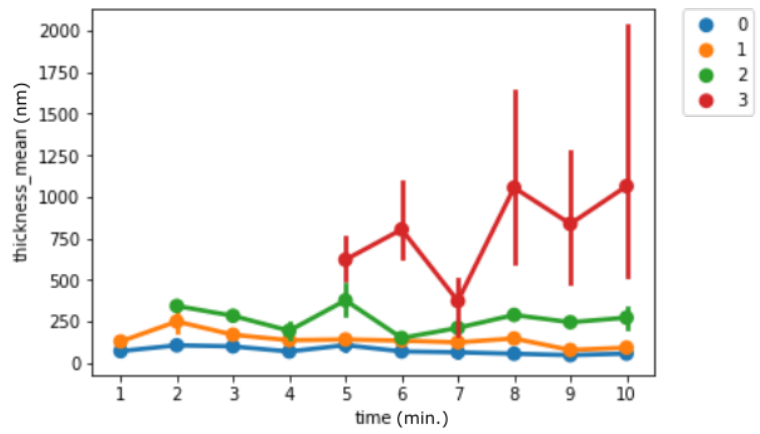

(b)

Figure 4. (a) Binary classification result by voltage from $10 \mathrm{~V}$ to $100 \mathrm{~V}$ (b) Binary classification result by time from $1 \mathrm{~min}$ to $10 \mathrm{~min}$ (line indicates $95 \% \mathrm{CI}$ ). 


\subsection{Classification Results}

\subsubsection{Prediction on Binary Classification}

Although AUC is our core measure, we report accuracy, precision, and recall measures together. As shown in Table 3, the AUC scores ranged between 0.93 and 1.00. A high AUC value greater than 0.8 denotes a reasonably good prediction rate and all the scores are above this value [37]. Compared to the other models, RF and GBT produced superior AUC scores. As mentioned in Section 3.2, the two algorithms are known as ensemble learning methods utilizing decision trees. The superiority of these models could be attributed to the following reasons. RF consists of a large number of individual decision trees that operated as an ensemble, in other words, the wisdom of crowds [27]. GBT model's key learning is from the previous mistakes [42]. It relies on the assumption that calculating the best next model combining with previous models, minimizes the overall prediction error that will lead to better performance. KNN is known to have advance in short execution time; however, it has shown to have the lowest accuracy for other classification problems [34].

Table 3. Comparison of evaluation metrics for classification algorithm on binary classification.

\begin{tabular}{ccccc}
\hline Algorithms & AUC & Accuracy & Precision & Recall \\
\hline LogReg & 0.98 & 0.90 & 0.88 & 0.90 \\
NB & 0.99 & 0.91 & 0.92 & 0.88 \\
KNN & 0.93 & 0.88 & 0.87 & 0.86 \\
SVM & 0.97 & 0.87 & 0.93 & 0.75 \\
DecTree & 0.91 & 0.92 & 0.94 & 0.88 \\
RF & 1.00 & 0.91 & 0.94 & 0.85 \\
Bagging & 0.97 & 0.90 & 0.96 & 0.90 \\
GBT & 1.00 & 0.93 & 0.94 & 0.90 \\
\hline
\end{tabular}

\subsubsection{Multiclass Classification}

Table 4 shows the evaluation metrics for multiclass classification. The macro precision scores ranged from 0.42 to 0.73 and macro recall scores ranged from 0.53 to 0.74 . For both cases DecTree has relatively higher scores than the other models. Considering accuracy, DecTree, Bagging, and GBT had relatively higher accuracy than the other models. LogReg, SVM, and KNN had relatively lower accuracy. The ensemble methods were superior as binary classification. DecTree is widely known to be powerful for classification because the tree tries to infer a split at each node.

Table 4. Comparison of evaluation metrics for classification algorithm on multiclass classification.

\begin{tabular}{cccccc}
\hline Algorithms & Accuracy & Micro Precision & Micro Recall & Macro Precision & Macro Recall \\
\hline LogReg & 0.74 & 0.74 & 0.74 & 0.42 & 0.53 \\
NB & 0.78 & 0.78 & 0.78 & 0.60 & 0.65 \\
KNN & 0.70 & 0.70 & 0.70 & 0.52 & 0.57 \\
SVM & 0.74 & 0.74 & 0.75 & 0.55 & 0.57 \\
DecTree & 0.82 & 0.84 & 0.84 & 0.73 & 0.74 \\
RF & 0.79 & 0.79 & 0.79 & 0.66 & 0.65 \\
Bagging & 0.81 & 0.80 & 0.77 & 0.70 & 0.65 \\
GBT & 0.80 & 0.80 & 0.80 & 0.63 & 0.69 \\
\hline
\end{tabular}

\section{Discussion}

There are several implications that we can achieve from the visualization (see Figure 2). First, we can detect if there is a correlation or trend among the features. For example, one can see that there is a positive increase as time and voltage increase. Second, we can also see that there are similar thicknesses generated from different settings. For example, the size of the circle is similar with the following settings: time (6 min) and voltage $(70 \mathrm{~V})$; 
time $(10 \mathrm{~min})$ and voltage $(90 \mathrm{~V})$. Even for the same thickness, there are certain levels of time or voltages that are preferred because of stability and also safety issues during the experiment. Third, if you want to determine the specific thickness, there is a minimal level that should be required. If we could predict that then several experiments could be saved. For example, to achieve thickness over 1000, a large amount of experiment settings on the left and lower sides was not needed. Therefore, we believe that if we have a large amount of experimental data, it could be narrowed into meaningful experimental setups.

Prediction scores for binary classification in this study show that our model is capable of predicting the presence of pores using the experimental levels of time and voltages. Researchers can predict whether pores will exist with the experimental settings. With the multiclass classification the evaluation scores were relatively lower. However, even with unbalanced and small sample size to predict four levels, over 0.7 for macro recall and precision shows the potential to move forward with the approach. Higher micro scores can be also interpreted; that is, certain levels have higher prediction scores. To obtain a reliable classification model even for small data sets, Beleites et al. recommended at least 75 to 100 samples per class to achieve a reasonable precision [43].

Overall, we can see that ensemble methods are superior for the binary classification problem. RF and GBT had a score of 1 for AUC. Ensemble methods are learning models based on the intuition that combining the opinions of multiple learners lead to better performance. RF uses a fully grown decision trees that have low bias, which are prone to overfitting. The idea is to resample the data multiple times and train a new classifier each time independently. In contrast, GBT adds a classifier each time, so that the next classifier is trained to improve the previous one. Both cases have shown good performance because they combine multiple individual models and show superior prediction power [44]. For multiclass classification, algorithms based on decision trees had relatively higher accuracy values. In our case, we had an imbalanced data set. A dataset is called imbalanced when the number of samples is different between each class. In this case the accuracy decreases for the minority classes. Decision tree algorithms are robust in these cases; therefore, we believe the DecTree algorithm and the ensembles (i.e., RF, bagging, GBT) also show relatively good performance. The KNN algorithm show the lowest performance for both binary and multiclass classifications. However, the score of 0.97 still shows a high performance for the binary classification problem. $\mathrm{KNN}$ is a robust and nonparametric machine learning algorithm. However, the algorithm depends greatly on the distances between points. If the classes overlap, the performance will decrease and we believe this may be the reason for relatively low performance [45]. Considering that this was the first study that comprised 100 samples and applied machine learning techniques for prediction, if we increase the sample size and get a better balanced dataset, we will get a more stable prediction on comparing the algorithms.

\section{Conclusions}

The present work demonstrates that the well-aligned formation of $\mathrm{TiO}_{2}$ nanostructures can be created in an ethylene glycol-based electrolyte by one-step anodization performed over various anodization voltages at anodization time ranging from $1 \mathrm{~min}$ to $10 \mathrm{~min}$. Anodization time and applied voltage have a great impact on the nanostructures of $\mathrm{TiO}_{2}$ and regularity of pore arrangement. With increasing applied voltage, the growth of oxide thickness increases. In addition, it was found that the oxide thickness changes its thickness in time with increasing tendency and decreasing tendency at specific anodization time and voltage. The highest values of oxide thickness and the regularity of the pore arrangement are observed at $100 \mathrm{~V}$ and $10 \mathrm{~min}$ as well. These consequences are described in terms of the blocking effect on the $\mathrm{TiO}_{2}$ surface caused by developed hydrolysis. On the above-obtained results, the ability to control the growth of $\mathrm{TiO}_{2}$ is an important factor towards a targeted development of Ti nanostructure because it is expected that a variety of nano-geometry industry exists. 
Therefore, we also applied machine learning to test the feasibility of predicting the surface aspects by the experimental settings, voltage and duration. We have observed that predicting the binary classification had higher accuracy than predicting the multiclass classification. However, the size of the dataset is relatively small than usual machine learning experiments. This is an innate limitation when applying machine learning techniques to experimental data for material science. We believe we have demonstrated classification capability to predict certain aspects from experimental results, which can help identify less useful experimental settings and properly use the cost and time to focus on specific experiments to achieve accurate results.

Author Contributions: Conceptualization, C.J. and S.-H.K.; methodology, C.J. and S.-H.K.; formal analysis, S.-H.K.; investigation, C.J.; resources, S.-H.K.; data curation, C.J.; writing, C.J. and S.H.K.; funding acquisition, S.-H.K. All authors have read and agreed to the published version of the manuscript.

Funding: This research was funded by National Research Foundation of Korea Grant, grant number NRF-2019R1C1C1005508 and Institute of Information \& communications Technology Planning \& Evaluation (IITP) grant funded by the Korea government (MSIT), grant number IITP-2020-0-01791.

Institutional Review Board Statement: Not applicable.

Informed Consent Statement: Not applicable.

Data Availability Statement: Data Sharing Not Applicable.

Conflicts of Interest: The authors declare no conflict of interest.

\section{References}

1. Byon, E.; Moon, S.; Cho, S.B.; Jeong, C.Y.; Jeong, Y.; Sul, Y.T. Electrochemical property and apatite formation of metal ion implanted titanium for medical implants. Surf. Coatings Technol. 2005, 200, 1018-1021. [CrossRef]

2. Fujishima, A.; Honda, K. Electrochemical photolysis of water at a semiconductor electrode. Nature 1972, 238, 37-38. [CrossRef] [PubMed]

3. Xie, Z.; Adams, S.; Blackwood, D.; Wang, J. The effects of anodization parameters on titania nanotube arrays and dye sensitized solar cells. Nanotechnology 2008, 19, 405701. [CrossRef]

4. Zhang, Y.; Li, X.; Hua, X.; Ma, N.; Chen, D.; Wang, H. Sunlight photocatalysis in coral-like TiO 2 film. Scr. Mater. 2009, 61, 296-299. [CrossRef]

5. Jeong, C.; Choi, C.H. Single-step direct fabrication of pillar-on-pore hybrid nanostructures in anodizing aluminum for superior superhydrophobic efficiency. Acs Appl. Mater. Interfaces 2012, 4, 842-848. [CrossRef]

6. Minagar, S.; Berndt, C.C.; Wang, J.; Ivanova, E.; Wen, C. A review of the application of anodization for the fabrication of nanotubes on metal implant surfaces. Acta Biomater. 2012, 8, 2875-2888. [CrossRef]

7. Smith, B.S.; Yoriya, S.; Johnson, T.; Popat, K.C. Dermal fibroblast and epidermal keratinocyte functionality on titania nanotube arrays. Acta Biomater. 2011, 7, 2686-2696. [CrossRef] [PubMed]

8. Yoo, S.Y.; Park, H.G. Effect of anodic oxidation process parameters on $\mathrm{TiO}_{2}$ nanotube formation in Ti-6Al-4V Alloys. Korean J. Met. Mater. 2019, 57, 521-528. [CrossRef]

9. Miao, Z.; Xu, D.; Ouyang, J.; Guo, G.; Zhao, X.; Tang, Y. Electrochemically induced sol- gel preparation of single-crystalline TiO 2 nanowires. Nano Lett. 2002, 2, 717-720. [CrossRef]

10. Sander, M.S.; Cote, M.J.; Gu, W.; Kile, B.M.; Tripp, C.P. Template-assisted fabrication of dense, aligned arrays of titania nanotubes with well-controlled dimensions on substrates. Adv. Mater. 2004, 16, 2052-2057. [CrossRef]

11. Jeong, C.; Ji, H. Systematic control of anodic aluminum oxide nanostructures for enhancing the superhydrophobicity of 5052 aluminum alloy. Materials 2019, 12, 3231. [CrossRef] [PubMed]

12. Ji, H.; Jeong, C. Study on corrosion and oxide growth behavior of anodized aluminum 5052 Alloy. J. Korean Inst. Surf. Eng. 2018, $51,372-380$.

13. Chen, $\mathrm{X}$.; Selloni, A. Introduction: titanium dioxide $\left(\mathrm{TiO}_{2}\right)$ nanomaterials. Chem. Rev. 2014, 114, 9281-9282. [CrossRef] [PubMed]

14. Lee, B.; Lee, S.; Choi, J.; Jeong, Y.; Oh, H.J.; Lee, O.Y.; Chi, C.S. Growth behaviors of anodic titanium oxide nanotubes in the ethylene glycol solution according to water contents. J. Korean Inst. Met. Mater. 2008, 46, 700-706.

15. Macák, J.M.; Tsuchiya, H.; Schmuki, P. High-aspect-ratio $\mathrm{TiO}_{2}$ nanotubes by anodization of titanium. Angew. Chem. Int. Ed. 2005, 44, 2100-2102. [CrossRef]

16. Diebold, U. The surface science of titanium dioxide. Surf. Sci. Rep. 2003, 48, 53-229. [CrossRef]

17. Henderson, M.A. A surface science perspective on $\mathrm{TiO}_{2}$ photocatalysis. Surf. Sci. Rep. 2011, 66, 185-297. [CrossRef]

18. Kukovecz, Á.; Kordás, K.; Kiss, J.; Kónya, Z. Atomic scale characterization and surface chemistry of metal modified titanate nanotubes and nanowires. Surf. Sci. Rep. 2016, 71, 473-546. [CrossRef] 
19. Jeong, C.; Lee, J.; Sheppard, K.; Choi, C.H. Air-impregnated nanoporous anodic aluminum oxide layers for enhancing the corrosion resistance of aluminum. Langmuir 2015, 31, 11040-11050. [CrossRef]

20. Kulkarni, M.; Mazare, A.; Schmuki, P.; Iglic, A. Influence of anodization parameters on morphology of $\mathrm{TiO}_{2}$ nanostructured surfaces. Adv. Mater. Lett. 2016, 7, 23-28. [CrossRef]

21. Alpaydin, E. Introduction to Machine Learning; MIT Press: Cambridge, MA, USA, 2020.

22. Liu, Y.; Zhao, T.; Ju, W.; Shi, S. Materials discovery and design using machine learning. J. Mater. 2017, 3, 159-177. [CrossRef]

23. Fan, R.E.; Chang, K.W.; Hsieh, C.J.; Wang, X.R.; Lin, C.J. LIBLINEAR: A library for large linear classification. J. Mach. Learn. Res. 2008, 9, 1871-1874.

24. Saritas, M.M.; Yasar, A. Performance analysis of ANN and Naive Bayes classification algorithm for data classification. Int. J. Intell. Syst. Appl. Eng. 2019, 7, 88-91. [CrossRef]

25. Nikam, S.S. A comparative study of classification techniques in data mining algorithms. Orient. J. Comput. Sci. Technol. 2015, 8, 13-19.

26. Chang, C.C.; Lin, C.J. LIBSVM: A library for support vector machines. ACM Trans. Intell. Syst. Technol. 2011, 2, 1-27. [CrossRef]

27. Liaw, A.; Wiener, M. Classification and regression by RandomForest. $R$ News 2002, 2, 18-22.

28. Becker, C.; Rigamonti, R.; Lepetit, V.; Fua, P. Supervised feature learning for curvilinear structure segmentation. In International Conference on Medical Image Computing and Computer-Assisted Intervention; Springer: Berlin/Heidelberg, Germany, 2013 ; pp. 526-533.

29. Natekin, A.; Knoll, A. Gradient boosting machines, a tutorial. Front. Neurorobot. 2013, 7, 21. [CrossRef]

30. Myles, A.J.; Feudale, R.N.; Liu, Y.; Woody, N.A.; Brown, S.D. An introduction to decision tree modeling. J. Chemom. J. Chemom. Soc. 2004, 18, 275-285. [CrossRef]

31. Brownlee, J. Master Machine Learning Algorithms: Discover how They Work and Implement Them from Scratch; Machine Learning Mastery: Cambridge, MA, USA, 2016.

32. Aly, M. Survey on multiclass classification methods. Neural Netw. 2005, 19, 1-9.

33. Bengio, Y.; Grandvalet, Y. No unbiased estimator of the variance of k-fold cross-validation. J. Mach. Learn. Res. 2004, 5, 1089-1105.

34. Liu, Y.; Bi, J.W.; Fan, Z.P. Multi-class sentiment classification: The experimental comparisons of feature selection and machine learning algorithms. Expert Syst. Appl. 2017, 80, 323-339. [CrossRef]

35. Powers, D.M. Evaluation: from precision, recall and F-measure to ROC, informedness, markedness and correlation. J. Mach. Learn. Technol. 2011, 2, 37-63.

36. Fawcett, T. ROC graphs: Notes and practical considerations for researchers. Mach. Learn. 2004, 31, 1-38.

37. Metz, C.E. Basic principles of ROC analysis. In Seminars in Nuclear Medicine; WB Saunders: Philadelphia, PA, USA, 1978; Volume 8, pp. 283-298.

38. Sokolova, M.; Lapalme, G. A systematic analysis of performance measures for classification tasks. Inf. Process. Manag. 2009, 45, 427-437. [CrossRef]

39. Su'ait, M.; Alamgir, F.; Scardi, P.; Ahmad, A. Morphological studies of vertical arrays $\mathrm{TiO}_{2}$ nanotubes by electrochemical anodization technique for dye sensitized solar cell application. Am. Inst. Phys. 2013, 1571, 835-842.

40. Zhang, A.Y.; Long, L.L.; Liu, C.; Li, W.W.; Yu, H.Q. Chemical recycling of the waste anodic electrolyte from the $\mathrm{TiO}_{2}$ nanotube preparation process to synthesize facet-controlled $\mathrm{TiO}_{2}$ single crystals as an efficient photocatalyst. Green Chem. 2014, 16, 27452753. [CrossRef]

41. Lee, K.C.; Sreekantan, S.; Ahmad, Z.A.; Saharudin, K.A.; Taib, M.A.A. Nucleation of octahedral titanate crystals using waste anodic electrolyte from the anodization of $\mathrm{TiO}_{2}$ nanotubes. CrystEngComm 2017, 19, 6406-6411. [CrossRef]

42. Frery, J.; Habrard, A.; Sebban, M.; Caelen, O.; He-Guelton, L. Efficient top rank optimization with gradient boosting for supervised anomaly detection. In Proceedings of the Joint European Conference on Machine Learning and Knowledge Discovery in Databases, Skopje, Macedonia, 18-22 September 2017; pp. 20-35.

43. Beleites, C.; Neugebauer, U.; Bocklitz, T.; Krafft, C.; Popp, J. Sample size planning for classification models. Anal. Chim. Acta 2013, 760, 25-33. [CrossRef]

44. Caruana, R.; Niculescu-Mizil, A. An empirical comparison of supervised learning algorithms. In Proceedings of the 23rd International Conference on Machine Learning, Pittsburgh, PA, USA, 25-29 June 2006; pp. 161-168.

45. Wettschereck, D.; Dietterich, T.G. An experimental comparison of the nearest-neighbor and nearest-hyperrectangle algorithms. Mach. Learn. 1995, 19, 5-27. [CrossRef] 